\title{
Bischof Eustathios im Paradies: Eine Kirche und ihr Stifter im kappadokischen Parnassos
}

\author{
Melih ARSLAN* - Philipp PILHOFER** - Yavuz YEĞİN****
}

\section{Parnassos: Eine Stadt und Ihre Erforschung}

Das antike Parnassos wird seit J.G.C. Anderson im heutigen Dorf Değirmenyolu (auch: Parlasan) im Bezirk Şereflikoçhisar in der Provinz Ankara lokalisiert. Nach antiken Kategorien befindet es sich im Nordwesten von Kappadokien (s. Karte), an der Grenze zu Galatien, zwischen Tatta Limne (Tuz Gölü) und Halys (Kızılırmak). ${ }^{1}$

Parnassos liegt an der berühmten Pilgerstraße, die von Konstantinopel über Chalkedon und Ankyra hier vorbeiführte und dann weiter über die Kilikische Pforte und die via Tauri nach Antiocheia am Orontes (und von dort weiter nach Jerusalem): Nach dem Itinerarium Burdigalense ist die mansio Parnasso die erste Station hinter der galatisch-kappadokischen Grenze. ${ }^{2}$ Ein Meilenstein, der von der Instandsetzung der Straße zeugt, hat sich vor Ort erhalten. ${ }^{3}$ Parnassos war damit - wie ganz Cappadocia - Teil der Dioecesis Pontica.

Spätestens Mitte des 3. Jahrhunderts gab es in der Region Christen, denn die Großeltern des späteren gotischen Bischofs Ulfila wurden in dieser Zeit als Christen aus der Region entführt. ${ }^{4}$

* Melih Arslan, Universität Hacı Bayram Veli, Ankara (arslanme lih06@hotmail.com; (1) https://orcid. org/0000-0002 -2152-1564).

** Dr. Philipp Pilhofer, Humboldt-Universität zu Berlin, Theologische Fakultät (philipp.pilhofer@huberlin.de; (1) https://orcid.org/0000-0003-4818-8017).

*** Dr. Yavuz Yeğin, Universität Ardahan, Abteilung für Archäologie, Ardahan (yavuz.yegin002@gmail. com; (1) https://orcid.org/0000-0001-7901-6352).

Wir danken Margherita Cassia (Catania), Jon Cubas Díaz (Göttingen), Susanne Froehlich (Greifswald), Stephen Mitchell (Berlin), Philipp Niewöhner (Göttingen) und Peter Pilhofer (Erlangen) für Diskussionen und Hinweise zu dieser Miszelle.

${ }^{1}$ Hild - Restle 1981, s.n. Parnas(s)os, 252-253; Cassia 2004, 107-108; Métivier 2005, 63.

${ }^{2}$ Itin. Burdig. 576,3. Siehe auch Itin. Anton. Aug. 144,1 und 206,3; vgl. Hild 1977, 35-41 (Route A1) und RRMAM 3.3, 263-277. Man beachte, daß Parnassos im Itinerarium Burdigalense nur als mansio bezeichnet wird, nicht als civitas (vgl. Métivier 2005, 281).

${ }^{3}$ RRMAM 3.3, 263-264, Nr. 158 (restituit); der Meilenstein ist abgebildet bei Hild 1977, Abb. 1.

${ }^{4}$ Zum frühen Christentum in Kappadokien generell vgl. die Beiträge von Margherita Cassia, Gaetano Arena und Aude Busine in Mitchell - Pilhofer 2019. Zur Episode um Ulfilas Großeltern vgl. Philostorgios,

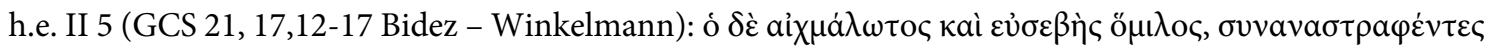

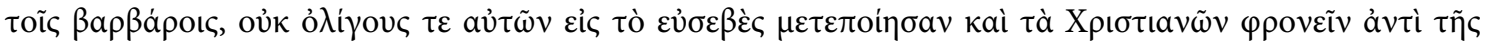

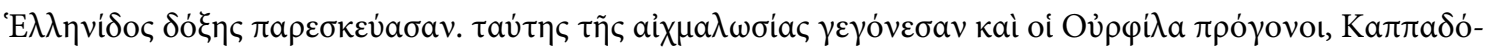

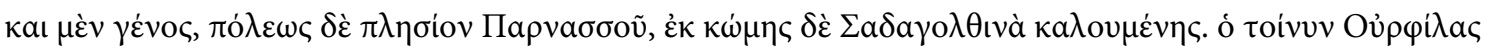

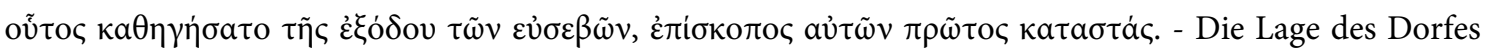




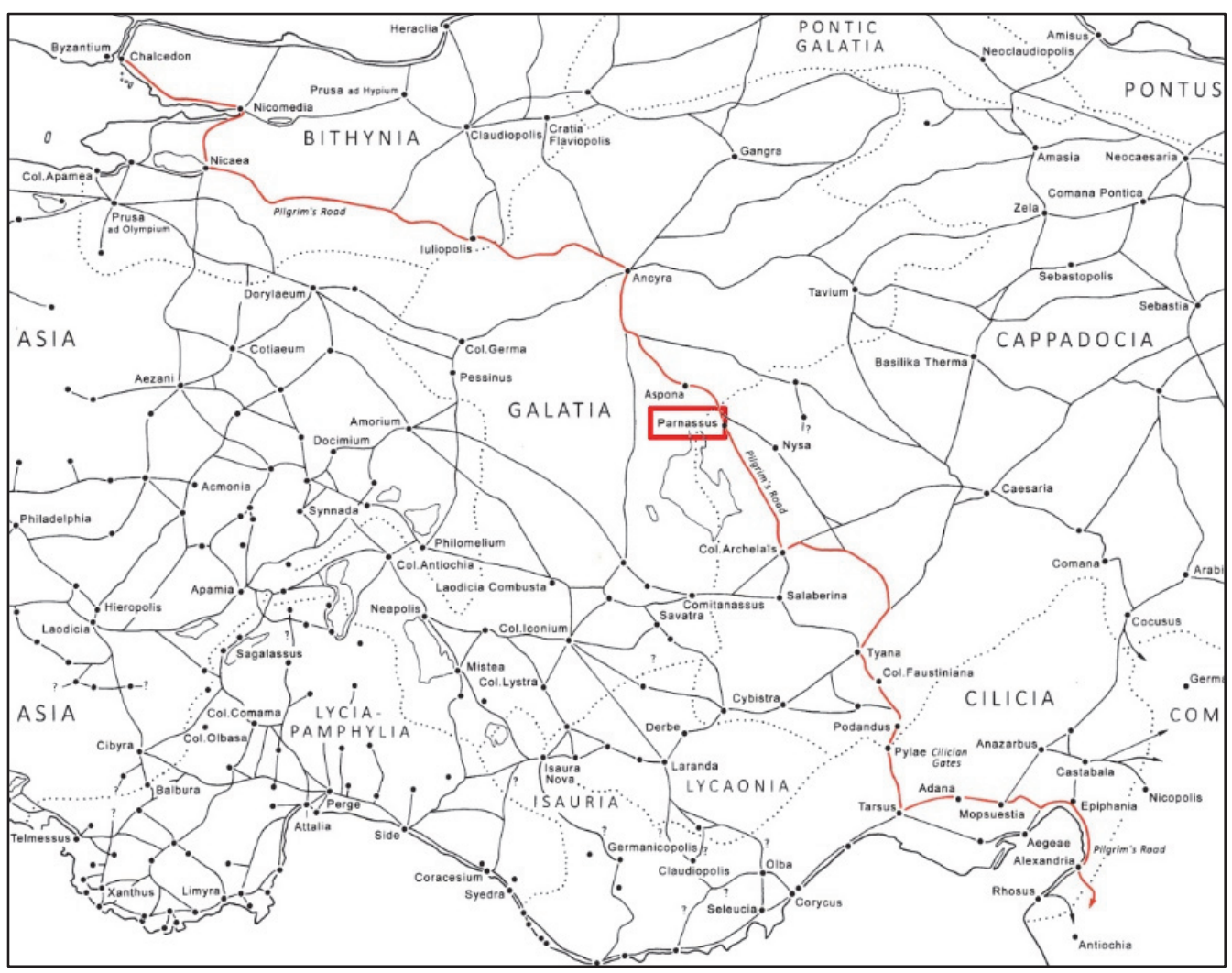

Karte: Das mittlere Kleinasien (Ausschnitt aus RRMAM 4.1, 85, Map 3)

Parnassos tritt womöglich bereits 325 in Nikaia als Bischofssitz in Erscheinung. ${ }^{5}$ Seitdem ist es immer wieder auf Synoden vertreten und wird auch in den Notitiae Episcopatuum von Konstantinopel bis in das 14. Jahrhundert als Bischofssitz geführt. ${ }^{6}$

Von 1991 bis 2007 ist in der Gegend durch Straßen- und Wasserleitungsbau und daran anschließend durch aufmerksame Schatzsucher vieles zerstört worden. Daher hat das Anadolu Medeniyetleri Müzesi Ankara („Museum für anatolische Zivilisationen“) eine Rettungsgrabung begonnen. Im Jahr 2010 hat der Direktor des Museums, Melih Arslan, mit seinem Team in Parnassos etwa für vier Monate ausgegraben. ${ }^{7}$ Durch die illegalen Grabungen waren Reste von Gräbern an den Tag gekommen. Die genehmigten Grabungen deckten ein reich mosaiziertes Gebäude auf. Dieses läßt sich mit der gebotenen Vorsicht (es scheint in einen bislang nicht erforschten Gebäudekomplex integriert zu sein) als Kirche klassifizieren.

Sadagolthina ist durch eine Inschrift gesichert: Hild - Restle 1981, s.n. Sadagolthina, 268, sowie Cassia 2004, 108-109.

${ }^{5}$ Gelzer - Hilgenfeld - Cuntz 1898, 74 Nr. 112 (die Überlieferung zu diesem Bischof ist aber sehr unsicher, s.u.).

${ }^{6} \mathrm{Zu}$ den Synoden vgl. die Angaben bei Hild - Restle 1981, 253. Da dort bei den ACO-Zitaten zwischen Band II und III das Zitierschema gewechselt wird, seien die Angaben zu ACO III hier noch einmal angegeben (sie betreffen alle Bischof Pelagios von Parnassos): ACO III 5 28,33 Nr. 43; 116,16 Nr. 46; 155,16 Nr. 27; 184,20 Nr. 41. Die Notitiae Episcopatuum: Darrouzès 1981, dort Notitiae $1^{411} ; 2^{480} ; 3^{341} ; 4^{432} ; 7^{510} ; 9^{394}$; $10^{463} ; 13^{464}$.

${ }^{7}$ Arslan - Sevim - Türkmen - Şirin 2012. 


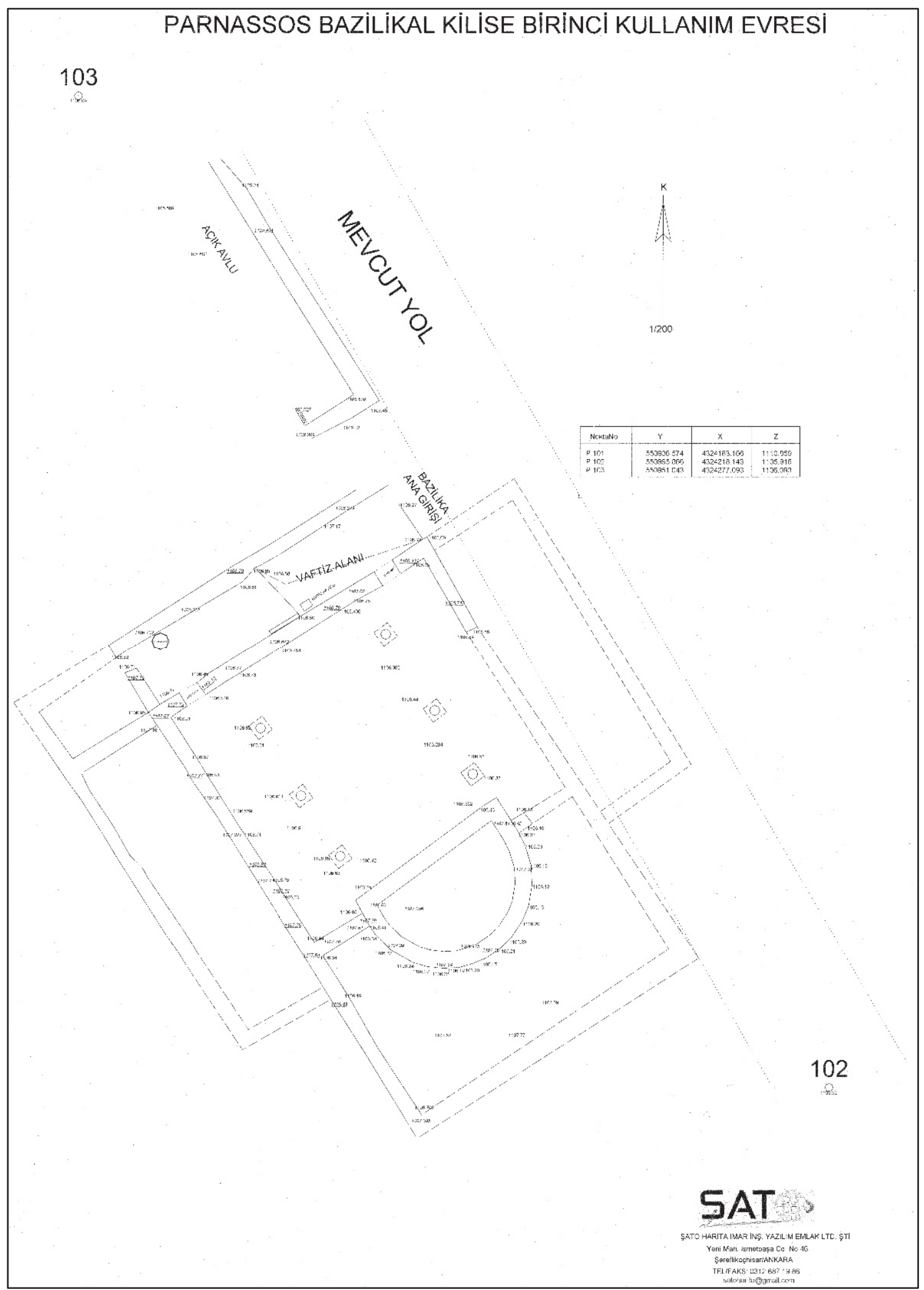

Plan 1) Die erste Bauphase 


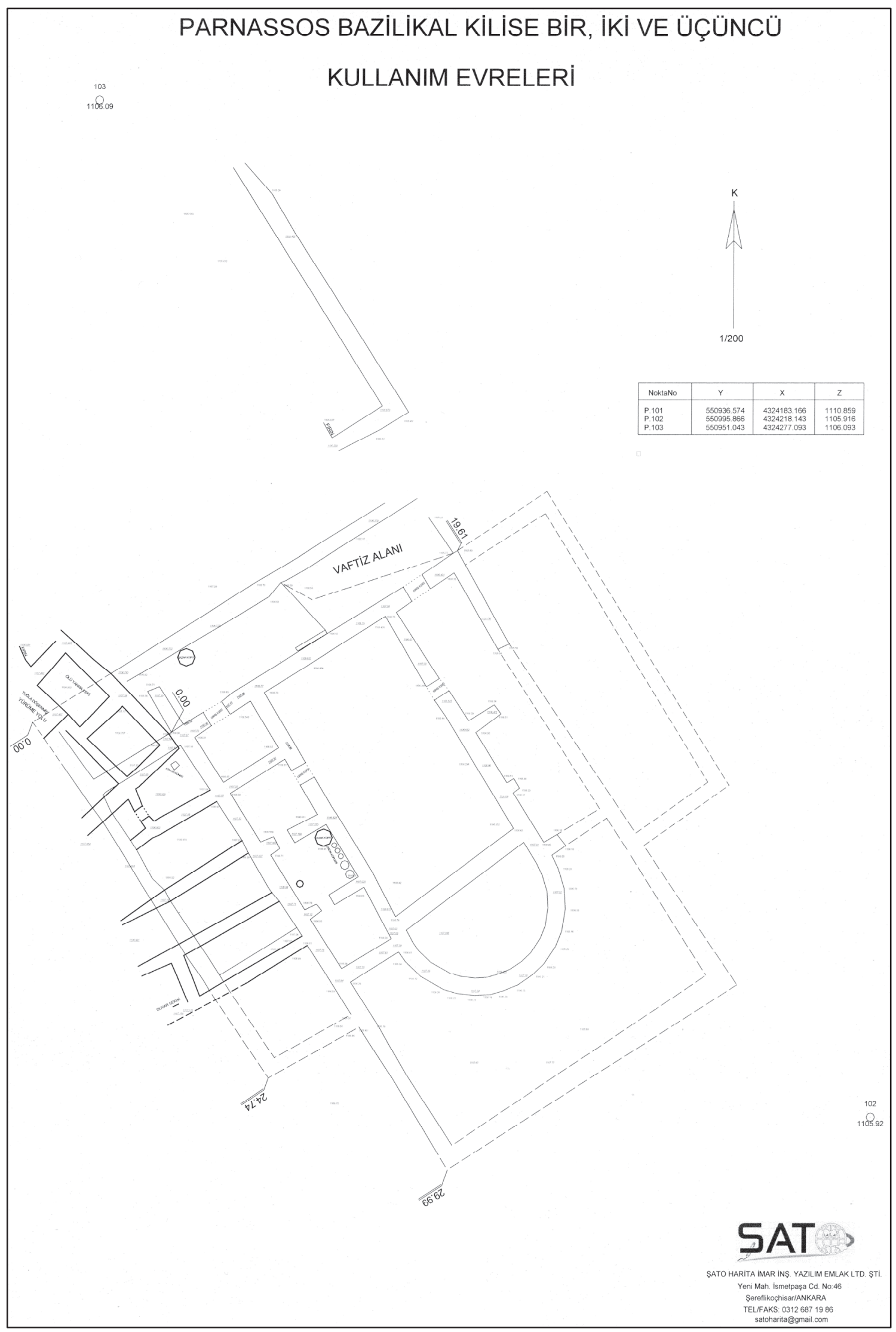

Plan 2) Die zweite Bauphase 


\section{Die Ausgrabungen}

Nach den Ausgrabungen läßt sich folgendes Bild zeichnen (oder zunächst auch betrachten: Abb. 1): Das in das abfallende Gelände hineingebaute und nach Süden ${ }^{8}$ ausgerichtete Gebäude besteht aus einem langrechteckigen Naos mit Apsis und verschiedenen seitlichen Nebenräumen; ursprünglich handelte es sich wohl um eine dreischiffige Basilika. Im Norden befand sich in ganzer Breite ein Narthex. Das Dach scheint eingestürzt zu sein, es wurden viele Dachziegel gefunden.

In der ersten Bauphase scheinen die Seitenschiffe jeweils durch eine drei Säulen umfassende Arkade vom Hauptschiff abgetrennt gewesen zu sein (s. Plan 1). Die drei Schiffe waren je durch eine Tür (die Schwellen sind erhalten) mit dem 3,90m breiten Narthex verbunden. Später wurden die Arkaden bis auf zwei Türen zum Naos zugemauert und die Seitenräume unterschiedlich unterteilt (s. Plan 2).

Das westliche Seitenschiff ist vollständig mosaiziert (geometrische Motive mit Diamanten in Kreisen; Perlenkette; Mäander). Später wurde es in vier ungefähr quadratische Räume geteilt. Der erste war nur vom Narthex aus zu betreten; der zweite vom verbliebenen Hauptsaal aus. Vom zweiten gelangte man in den dritten und nur von dort in den vierten. Im dritten Raum scheinen u.a. Amphoren gelagert worden zu sein.

Die östlichen Räume sind weitgehend zerstört. Hier fanden in den frühen 90er Jahren nach Berichten illegale Grabungen statt, bei denen gewölbte Grabstrukturen ausgeraubt wurden. Diese scheinen mit dicken Marmorplatten bedeckt gewesen zu sein. Die bei den späteren Grabungen gefundenen Knochenreste aus diesem Gebiet waren laut den Anthropologen des Museums aber keine menschlichen Überreste. Es wurden hier viele Tesserae gefunden, die darauf hindeuten, daß auch die östlichen Räume mosaiziert waren.

Die Apsis hat einen Radius von 5,20m. Sie scheint vollständig von Mosaiken bedeckt gewesen zu sein. Da die Apsis aber $90 \mathrm{~cm}$ höher (und damit näher an der Oberfläche) liegt als der Naos, sind diese Mosaiken jedoch völlig zerstört.

Das Hauptschiff, später zum zentralen langrechteckige Raum umgebaut, mißt etwa $15 \mathrm{~m}$ mal 8,5m. Der ganze Raum ist von einem gut gearbeiteten Mosaik bedeckt, das allerdings durch den Bau einer Wasserleitung sowie die Wurzeln von Pflanzen stellenweise beschädigt worden ist. Die Tesserae sind sauber geschnitten und $1 \times 1 \mathrm{~cm}$ groß. Es wurden schwarze, weiße, grüne, rote, gelbe, blaue und braune Steine verwendet.

Das Mosaik (s. Abb. 2) hat einen breiten dreifachen Rahmen, der von außen nach innen folgende Elemente enthält: zuerst geometrische Motive, dann Palmetten und schließlich Akanthusblätter, die mit einem Spiralmotiv begrenzt sind. Von diesem Rahmen umgeben ist das Hauptmotiv, ein sogenannter „Figurenteppich“. Auf hellem Grund sind in einem regelmäßigen Muster Blumen aus roten Steinen mit zwei Blättern als Füllmotiv verwendet worden; dazwischen und sehr viel größer sind verschiedene Tiere und vier unterschiedliche Baum-Arten abgebildet. Die Tiere sind der Reihe nach: Leopard, Strauß, Schaf, Kaninchen, Bergziege, Reh, Fohlen, Vogel, Pferd, Ziege, Hirsch (s. Abb. 3), Ente, Stier. Diese Motivik könnte ganz neutral als geometrisch-vegetabile Tierdarstellung aufgefaßt werden - oder als Darstellung des paradiesischen Tierfriedens (die

\footnotetext{
${ }^{8} \mathrm{Ob}$ das eine beabsichtigte Ausrichtung nach Jerusalem bedeutet, oder ob die Ausrichtung durch die umgebende Bebauung vorgegeben war, muß offen bleiben.
} 
Diskussion folgt sogleich). Mittig im Raum und damit mitten im „Paradies“ ist ein Medaillon zu sehen, das eine Inschrift enthält (s. den folgenden Abschnitt).

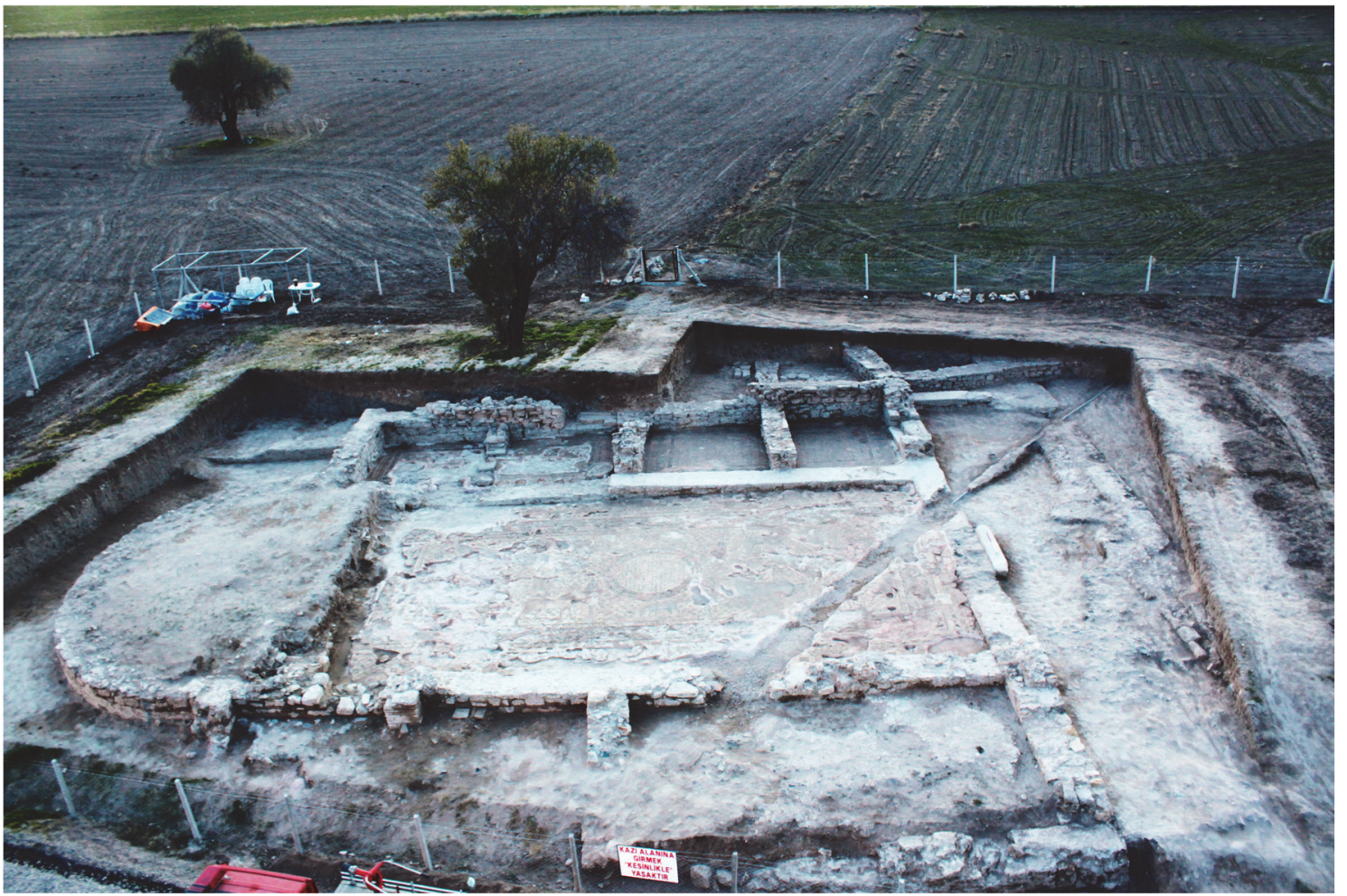

Abb. 1) Übersicht der Ausgrabung

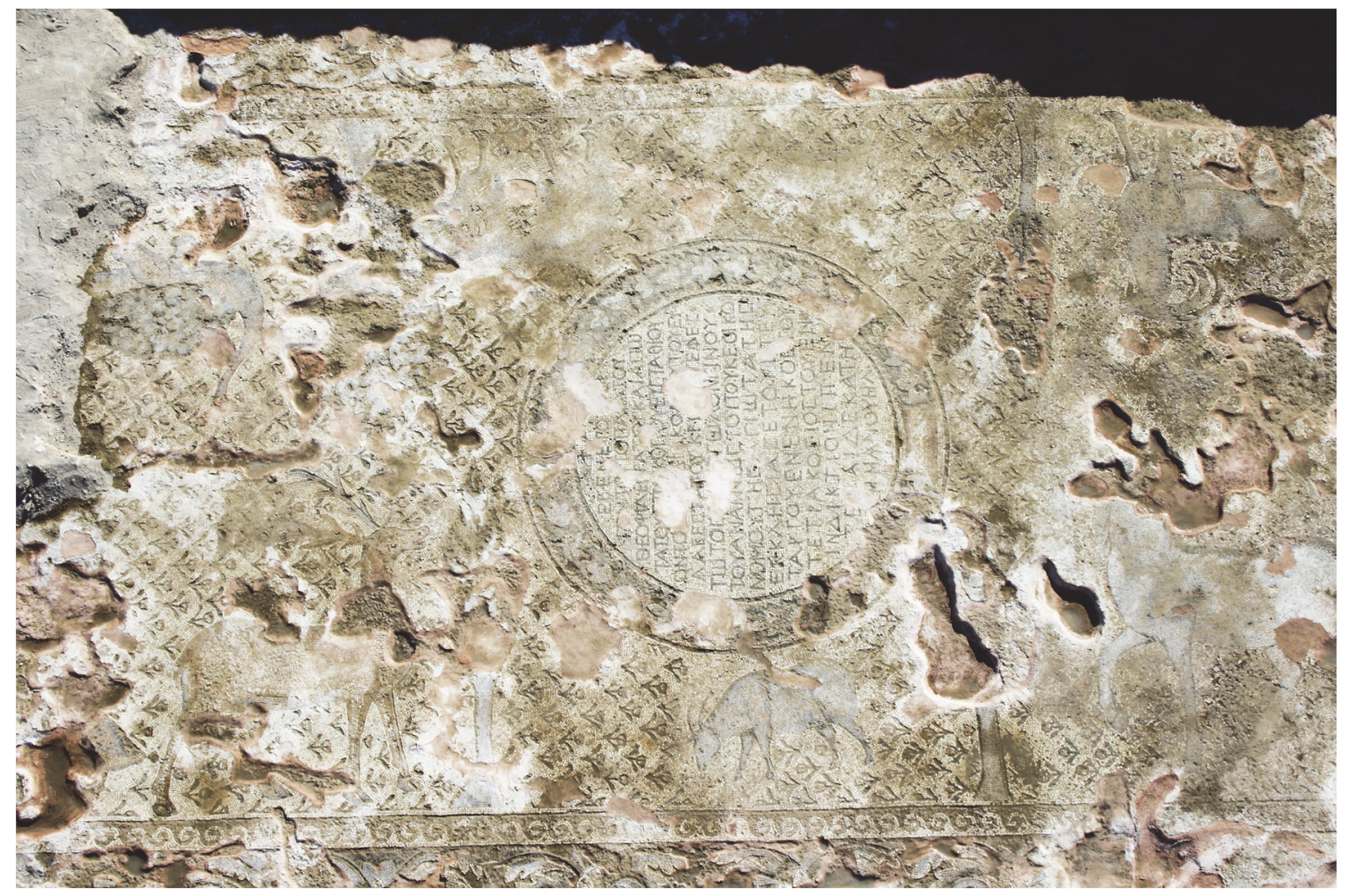

Abb. 2) Das Mosaik im Hauptschiff, Gesamtansicht 


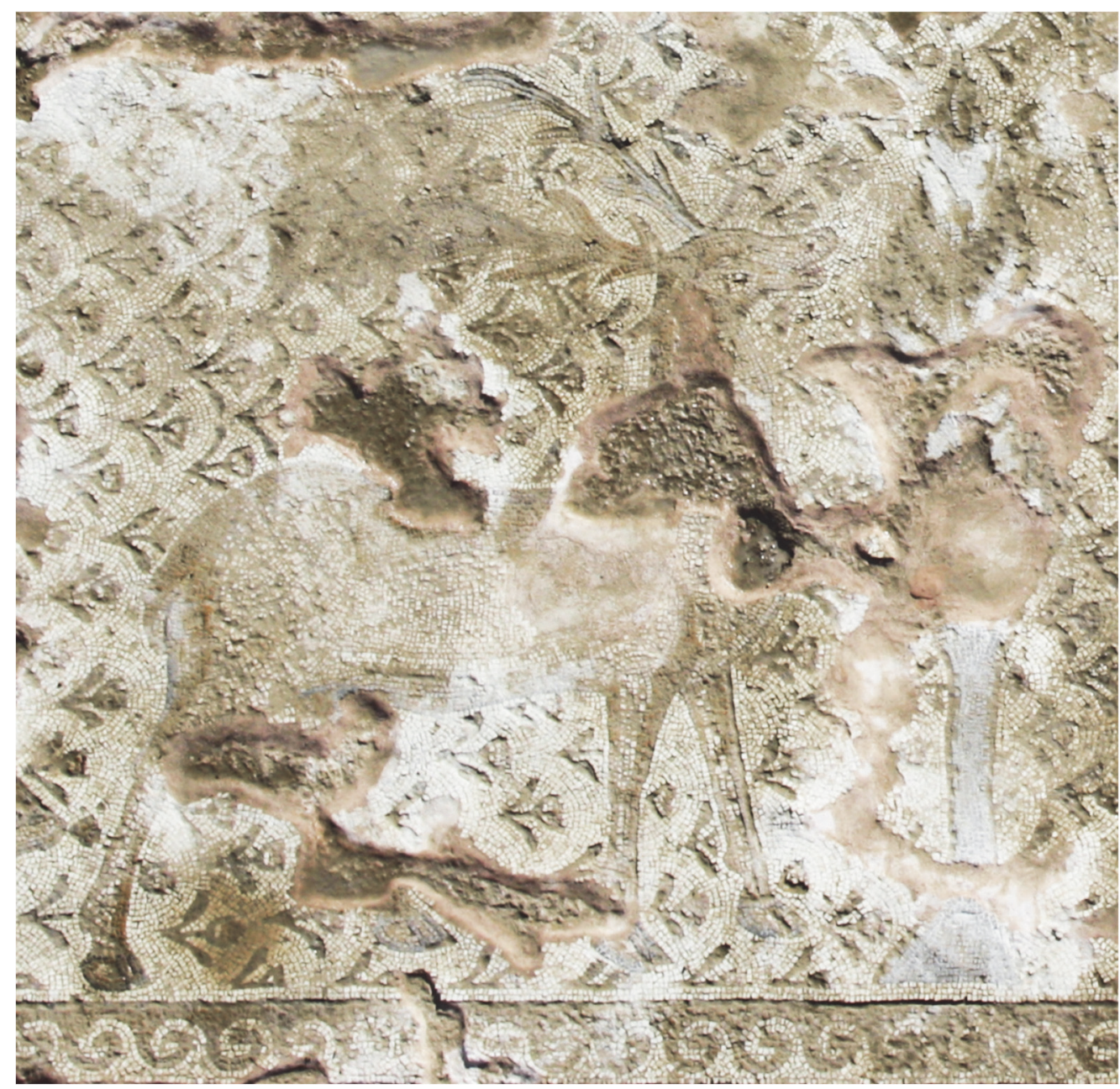

Abb. 3) Detail des Mosaiks: Mäander-Muster am Rand, Hirsch mit Baum auf hellem Blumen-Hintergrund

Bei den Ausgrabungen sind nur wenige Kleinfunde ans Licht gekommen. Keramikscherben von alltäglichem Gerät sind in spätantike Zeit zu datieren. Zudem sind mehrere Münzen gefunden worden, die von den Numismatikern des Museums, Sena Mutlu und Ülkü Devecioğlu, untersucht wurden. Eine silberne Münze läßt sich in die Zeit des Kaisers Galerius datieren (also 305-311), sie wurde in Nikomedia (heute İzmit) geprägt. Zwei weitere Münzen stammen aus den Regierungszeiten von Konstantin II. (337-340) und wahrscheinlich Theodosios II. (408-450).

Eine genaue Untersuchung der Mosaiken steht noch aus, ${ }^{9}$ allerdings ist schon angesichts des Gebäudes, in dem sich das Mosaik befindet, nicht davon auszugehen, daß es als neutrale Tierdarstellung wahrgenommen wurde. Abbildungen des Tierfriedens auf Mosaiken waren im benachbarten syro-kilikischen Raum sehr beliebt: „Charakteristisches Thema auf Mosaikböden nach der Mitte des 5. J[ahr]h[undert]s sind die sog[enannten] Paradiesdarstellungen. Wilde u[nd] zahme Tiere stehen in einer von Bäumen $\mathrm{u}[\mathrm{nd}]$ Blumen symbolisierten ,paradiesischen' Landschaft friedlich nebeneinander. ${ }^{\text {"10 }}$ Eine enge Parallele bildet das etwas weiter südlich entlang der Pilgerstraße in

\footnotetext{
${ }^{9}$ Eine separate und detaillierte Aufarbeitung der Mosaiken befindet sich in Vorbereitung.

${ }^{10}$ Hild - Hellenkemper - Hellenkemper-Salies 1990, 336; zur Besprechung zahlreicher Beispiele s. dort, 336-347 (inkl. einiger Abbildungen). Auch Wisskirchen 2009 diskutiert das Motiv und bietet dabei elf spätantike Beispiele mit Abbildungen (davon nur drei nicht aus dem syro-kilikischen Raum: 5x Kilikien, 4x Syrien, 2x Jordanien, 1x Korsika; Motive ohne Raubtiere wie das „Paradies von Dağ Pazarı“ nimmt sie nicht
} 
einem nicht sicher zugeordneten Gebäude in Mopsouhestia befindliche zentrale Mosaik; nur mit dem Unterschied, daß sich dort in der Mitte nicht ein Medaillon mit der Stifterinschrift, sondern die Arche Noah befindet. ${ }^{11}$ In ähnlichen Fällen wird durch Inschriften der entsprechende JesajaText explizit zitiert, etwa in Korykos oder Anemourion. ${ }^{12}$

So eindeutig ist der Bezug in Parnassos nicht. Insgesamt scheint es uns aber plausibel, daß die meisten Personen in der Spätantike, die dieses Mosaik mit seiner friedfertigen Darstellung von unterschiedlichen Tieren in einem Blumenmeer und zwischen Bäumen erblickten, hier den paradiesischen Tierfrieden erkannten; dies eben auch ohne expliziten Hinweis durch ein Bibelzitat.

Mit der Pantaleon-Kirche im kilikischen Aphrodisias existiert ein ähnlich großer und ähnlich strukturierter Bau - sofern die These von Hild und Hellenkemper zutrifft, daß die äußere Apsis dort nur die Apsis des Umgangsraumes war und die eigentliche Apsis nicht ergraben wurde. ${ }^{13}$ Auch in Aphrodisias sind reiche Mosaiken erhalten, samt einer zentral angebrachten Stifterinschrift. Der Bau in Aphrodisias wird von Hild und Hellenkemper dem fortgeschrittenen 5. Jahrhundert zugeordnet. Da in Parnassos nur eine Rettungsgrabung stattfand, konnten die umgebenden Gebäudereste nicht genauer untersucht werden; so muß offen bleiben, ob die ergrabenen Räume möglicherweise - wie in Aphrodisias - Teil eines größeren Komplexes waren.

Insgesamt handelt es sich bei der Kirche von Parnassos also vermutlich um eines der in Kappadokien nicht allzu zahlreichen Beispiele für eine dreischiffige Basilika. ${ }^{14}$ Diese wurde wohl später durch Abtrennung der Seitenschiffe in eine Saalkirche umgewandelt. Darin ähnelt sie zwei Kirchen in der Region, nämlich einmal der etwas weiter südlich an der Pilgerstraße gelegenen sog. Konstantinsbasilika von Andabalis (türkisch: früher Eski Andaval, heute treffenderweise Yeniköy), die auch ähnlich groß ist, sowie der „Kirche II“ von Mokisos. ${ }^{15}$ Von großer Bedeutung und besonderem Wert sind jedoch vor allem die Mosaikfunde, da aus Kappadokien bisher zwar viele Malereien bekannt sind, nicht jedoch Mosaiken; insbesondere nicht solche aus der Spätantike. ${ }^{16}$ Die Motivik findet sich bislang vor allem in der Dioecesis Orientis, nicht aber im Norden und Westen Kleinasiens.

in de Blick) sowie einer sehr kleinteiligen Kategorisierung. Wisskirchen bietet auch eine Diskussion der relevanten Bibel- und Kirchenväter-Texte. Vgl. jüngst Patacı 2016.

${ }^{11}$ Budde 1969, passim. Es ist bis heute unklar, ob es sich um eine Kirche oder eine Synagoge handelt (vgl. IJO II, 496, Anm. 19).

${ }^{12}$ So etwa in der „Kathedrale“ von Korykos (MAMA II, 106f.) oder in der Nekropolen-Kirche von Anemourion (Russell 1987, 70-74, Nr. 14).

${ }^{13}$ So Hild - Hellenkemper 1990, 195 als Modifiaktion zu Budde 1987.

${ }^{14}$ Restle 1979 kommt in seinem Katalog (34-48) auf sechs Exemplare.

${ }^{15}$ Zur im Winter 1976/77 eingestürzten „Konstantinsbasilika“ vgl. Restle 1979, 36-42, mit Abbildungen, Grundriß und Rekonstruktionen; laut Grundriß maß das Mittelschiff etwa 6x10m. Zur „Kirche II“ von Mokisos s. ebd., 46-48 (mit Plan und Abbildungen).

${ }^{16}$ Im ganzen RBK-Artikel (Restle 1978) werden Mosaiken nicht einmal erwähnt, während die Malereien über beinahe 40 Spalten besprochen werden. 


\section{Die Inschrift}

Auf dem Mosaik ist zentral ein großes Medaillon mit einem Durchmesser von etwa 2,40m abgebildet (s. Abb. 4). Das Medaillon besteht aus einem Rahmen und einem Hauptfeld. Der etwa $30 \mathrm{~cm}$ breite äußere Rahmen ist mit Weinblättern geschmückt und hat an der dem Eingang zugewandten Seite eine Fläche, die von den Ausgräbern als „Trittfläche zum Lesen der Inschrift“ interpretiert wurde. ${ }^{17}$ Das zentrale Feld hat einen Durchmesser von $1,80 \mathrm{~m}$ und einen hellen Hintergrund; darauf ist mit dunklen Tesserae die Stiftungsinschrift eingebracht. Die Buchstaben stehen im oberen Drittel gedrängt, darunter mit etwas mehr, z.T. auch mit sehr viel Zwischenraum. Die Schrift ist so ausgerichtet, daß eine eintretende Person sie lesen kann.

Treten wir also ein: ${ }^{18}$

fol. Kreuz fol.

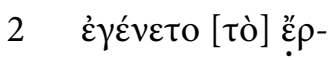

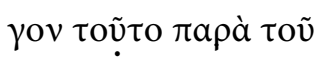

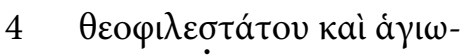

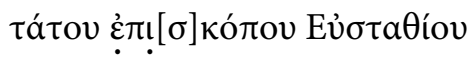

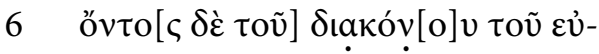
$\lambda \alpha \beta \varepsilon \sigma \tau a \dot{\tau} \tau o v \mathrm{~K} \rho \dot{i}[\sigma \pi \mathrm{\sigma o}] \mathrm{\varepsilon} \varphi \varepsilon \varepsilon \sigma-$

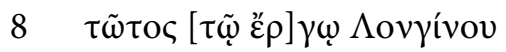

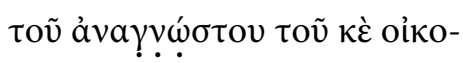

10 vó

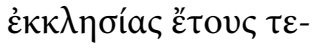

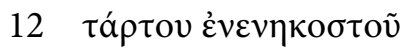

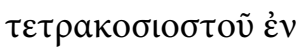

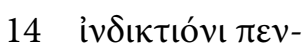

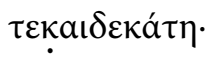

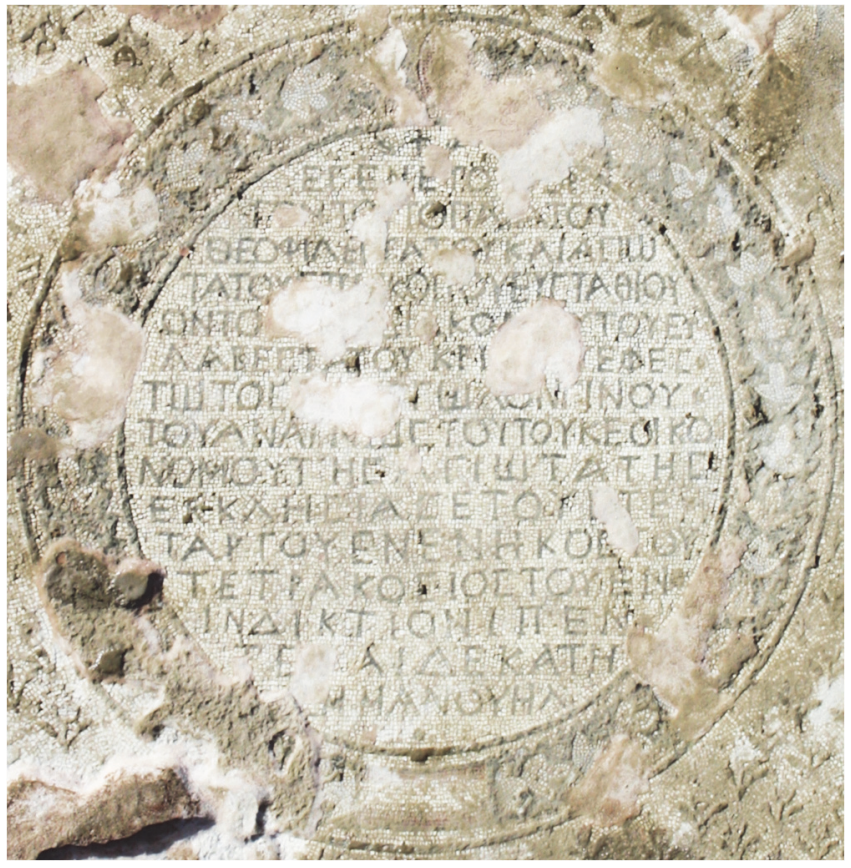

Abb. 4) Detail des Mosaiks: Die Inschrift im Medaillon

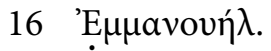

\section{Anmerkungen zum Text}

Z. 1: Vor der Lücke ist der Beginn der Querhaste des Tau noch zu erkennen, daher ist die Ergänzung [ [ó] recht sicher.

\footnotetext{
${ }^{17}$ Arslan - Sevim - Türkmen - Şirin 2012, 196: „Daire şeklindeki yazıtın bordür kenarına, üstüne basıp okuyabilmeleri için giriş istikametinde bir de ayak yeri yapılmıştır.“

${ }^{18}$ Eine vorläufige türkische Übersetzung (basierend auf Vorarbeiten von Thomas Drew Bear) ist schon in Arslan - Sevim - Türkmen - Şirin 2012, 196 publiziert worden. Von den dortigen Abbildungen wurde die Inschrift im SEG übernommen: SEG LXIV, 1502.
} 


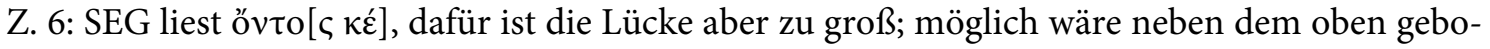

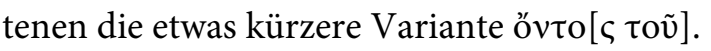

Z. 7: SEG liest vor der Lücke KP $\Omega$, allerdings ist auf besseren Abbildungen sicher zu erkennen, daß der letzte Buchstabe vor der Lücke ein Iota ist. Der Name Krispos ist nur exempli gratia zu verstehen, allerdings gibt es nicht allzu viele Alternativen ähnlicher Länge, die den Genitiv auf ov bilden und in dieser Zeit in Kleinasien in Gebrauch waren.

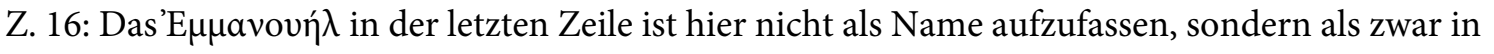
griechischen Buchstaben, aber auf Hebräisch formulierten Wunsch: „Gott (sei) mit uns“. In ver-

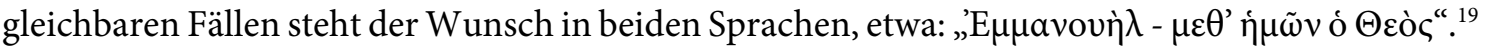

\section{Übersetzung}

„Dieses Werk entstand auf Anordnung des gottgeliebtesten und heiligsten Bischofs Eustathios, als aber der frömmste Krispos Diakon war, unter der Bauaufsicht des Longinos, des Vorlesers und Verwalters der heiligsten Kirche, im vierhundertvierundneuzigsten Jahr in der fünfzehnten Indiktion; Gott mit uns!“

\section{Datierung}

Mit den ausgegrabenen Münzen aus dem frühen 4. Jahrhundert läßt sich eine Frühdatierung des Mosaiks nicht begründen: Um auf eine Jahreszahl vor 350 n.Chr. zu gelangen, müßte man den Beginn der Ära in das 2. Jahrhundert vor Christus legen. Ein so frühes im spätantiken Kappadokien relevantes ärabegründendes Datum ist nahezu ausgeschlossen. Auch spricht die Gestaltung des Mosaiks selbst dagegen (Details wird hier allerdings erst eine genauere Analyse desselben bringen).

Die Datierung in das 494. Jahr und die 15. Indiktion in der Inschrift selbst werfen die Frage auf, auf welche Ära sich die Jahreszahl bezieht. ${ }^{20}$ In der ersten Bekanntmachung der Inschrift sowie im SEG wird mit dem Jahr 469/70 n.Chr. gerechnet, also wohl die galatische Ära vorausgesetzt (die mit der Provinzgründung 25 v.Chr. beginnt). Allerdings fällt das Jahr 469/70 n.Chr. nicht in eine 15. Indiktion; auch ist es fraglich, inwiefern die galatische Ära für Kappadokier von Interesse sein sollte, zumal in so später Zeit.

In hellenistischer Zeit sowie in der Kaiserzeit scheint in Kappadokien vor allem die Datierung nach Regierungsjahren des Herrschers verbreitet gewesen zu sein. ${ }^{21}$ Das allerdings ist in unserem Fall angesichts der Jahreszahl ausgeschlossen. Eine eigene Provinzära scheint in Kappadokien nicht verbreitet gewesen zu sein; eine lokale Ära aus Parnassos ist bisher nicht bekannt. Möglich wäre, daß - wie auch in einigen anderen kleinasiatischen Gebieten - die aktische Ära verwendet wurde, die sich auf den Sieg Octavians bei Actium bezieht: Diese Ära scheint im frühen 1. Jahrhundert im westlichen Kleinasien verbreitet gewesen zu sein; zumindest in Philadelpheia läßt sie

\footnotetext{
${ }^{19}$ Zwei Beispiele aus Phrygien: ICG 1462 (= MAMA XI, Nr. 166) und 1702 (= MAMA I, Nr. 397).

${ }^{20}$ Generell ist aber festzuhalten, daß auch im 6. Jahrhundert noch gelegentlich nach den Ären, die ihr Epochenjahr rund um die Zeitenwende haben, datiert wird. Vgl. etwa aus Anazarbos die Datiertung in das 535. Jahr der Ära der Stadt: IK 56, Nr. 62 (= 516 n.Chr.).

${ }^{21}$ Vgl. Leschhorn 1993, 18-19, der Belege bis Gordian III. bietet.
} 
sich noch im 6. Jahrhundert nachweisen. ${ }^{22}$ Im mittleren und östlichen Kleinasien ist sie bislang allerdings nicht belegt, dafür aber wiederum in Syrien (z.B. in Antiocheia und Laodikeia). ${ }^{23}$ Kappadokien war zu Lebzeiten des Augustus gar nicht direkt unter römischer Kontrolle. Allerdings hat wahrscheinlich der kappadokische König Archelaos I. die Stadt Mazaka zu Ehren des Augustus als Kaisareia benannt. ${ }^{24}$ Es gäbe also durchaus Anknüpfungspunkte.

Für die aktische Ära waren regional zwei unterschiedliche Epochenjahre im Gebrauch, entweder 31/30 oder 32/31 v. Chr. ${ }^{25}$ Wenn man letzteren Termin annähme, käme man für unsere Inschrift auf das Jahr 462/63. Das Jahr 462 fiele in eine 15. Indiktion und würde daher zur Inschrift passen.

\section{Identifikation des Bischofs}

Während der Name des Diakons aufgrund der Lücke ohnehin unsicher ist und der Name des Vorlesers und Verwalters Longinos sehr viel zu häufig ist, um auf dieser Hierarchieebene eine realistische Identifikation vorzuschlagen (und es ohnehin keine weiteren Belege aus Parnassos gibt), ist die Datenlage beim Bischof Eustathios sehr viel besser. Aus Parnassos sind bislang zwei oder drei Bischöfe mit dem Namen Eustathios belegt. Der älteste ist der oben schon erwähnte, aber unsicher überlieferte Vertreter auf dem Konzil von Nikaia $325 .{ }^{26}$ Der jüngste lebte am Ende des 7. Jahrhunderts und unterschrieb am Quinisextum. ${ }^{27}$

Zwischen diesen beiden ist Mitte des 5. Jahrhunderts ein Bischof Eustathios von Parnassos mehrfach und daher sehr gut belegt (wahrscheinlich ist er zugleich auch das Vorbild, nach dem der Name in der Liste von 325 ergänzt wurde). Er war auf den Konzilien 431 in Ephesos ${ }^{28}$ und 451 in Chalkedon ${ }^{29}$ anwesend. Außerdem ist er Ende der 450er Jahre als Unterzeichner zweier Schriften belegt, er unterschrieb nämlich einerseits die briefliche Stellungnahme seiner Provinz in Sachen

${ }^{22}$ Buckler 1917, Nr. 8 und 9. Zur Verbreitung der aktischen Ära vgl. Leschhorn 1993, 425-428.

${ }^{23} \mathrm{Zu}$ den syrischen Städten vgl. Leschhorn 1993, 226 mit Anm. 9 und 10.

${ }^{24}$ Das zumindest scheint die weithin akzeptierte These zu sein (vgl. etwa Hild - Restle 1981, 63); die Quellenlage ist allerdings nicht eindeutig, die Belege bietet Cassia 2004, 160-161.

${ }^{25} \mathrm{Zu}$ den Details vgl. Leschhorn 1993, 225-228.

${ }^{26}$ Gelzer - Hilgenfeld - Cuntz 1898, 74, Nr. 112; mit guten Gründen vermutet allerdings Honigmann, daß dieser Eintrag eine spätere Ergänzung ist (Honigmann 1939, 52-56, insbesondere 55, Nr. 79), Parnassos wäre also auf dem Konzil nicht vertreten gewesen. Schäferdiek 1979, 141-143 (= 287-289) wiederum kann zeigen, daß Honigmanns umfassende Ergänzungs-These zumindest nicht in allen Teilen gültig ist.

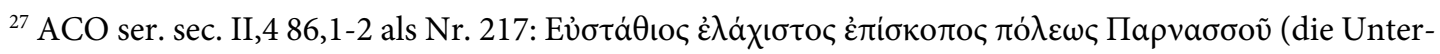
schrift zeigt ein ganz anderes bischöfliches Selbstbild als unser Mosaik).

${ }^{28}$ Eustathios war nicht nur 431 auf dem Konzil anwesend, sondern auch in die zahl- und wendungsreichen Streitigkeiten in den Sommermonaten involviert. Hier ist nicht der Ort, um dies im Einzelnen nachzuzeichnen; wir geben aber sämtliche recherchierten Belegstellen für unseren Eustathios an (zum Teil handelt es sich nur um Übersetzungen bzw. dieselbe Unterschrift in zwei unterschiedlichen Quellensammlungen): ACO I 1,3 25,20 Nr. 23; 27,4 Nr. 22; I 1,5 124,4 Nr. 38; I 2 83,5 Nr. 23; I 3108 Nr. 23; I 4 29, 18 Nr. XLVI; 38 Nr. 42 ; 46 Nr. 41 („Myrinae“ Fehler für Parnassos, vgl. Price - Graumann 2020, 351, Anm. 143); 243 Nr. 36.

${ }^{29}$ ACO II 1,1 150,17 Nr. 13 (in der lateinischen Fassung: ACO II 3,1 134,11 Nr. 13) bietet die Unter-

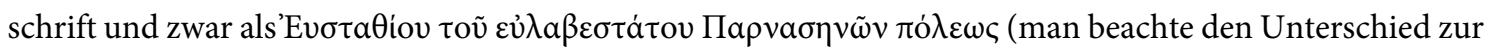
Titulatur in der Inschrift). 
Timotheos Ailuros an Kaiser Leon I. ${ }^{30}$ sowie andererseits die Epistula encyclica des Gennadios von Konstantinopel. ${ }^{31}$ Zwar sind keine theologischen Traktate von ihm selbst erhalten, aber zumindest eine wahrscheinlich gegen ihn persönlich gerichtete Antilogie, nämlich die des Eutherios von Tyana. ${ }^{32}$

Wenn nun die oben vorgenommene Datierung des Mosaiks auf das Jahr 462 zutrifft, ${ }^{33}$ dann ist es sehr plausibel, daß der gerade diskutierte Bischof Eustathios von Parnassos noch mindestens bis in die frühen 460er Jahre lebte und also mit dem Erbauer des Gebäudes gleichzusetzen ist. Eustathios wäre also ein Mann, der als eher junger Bischof auf dem Konzil von Ephesos 431 teilgenommen hat, im mittleren Alter in Chalkedon 451 aktiv mitwirkte und dann in einem für antike Verhältnisse schon hohen Alter in seiner Stadt eine Kirche errichten ließ.

\section{Bibliographie}

\section{$\mathrm{ACO}$}

Arslan - Sevim - Türkmen

- Şirin 2012

Buckler 1917

Budde 1969

Darrouzès 1981
Budde 1987

Cassia 2004

Acta Conciliorum Oecumenicorum, 1927-2016.

M. Arslan - M. Sevim - M. Türkmen - A. Şirin, Parnassos antik kenti 2010 yılı kilise kurtarma kazısı, in: A. Özme (ed.), 20. Müze çalışmaları ve kurtarma kazıları sempozyumu, 25-29 Nisan 2011, Bodrum, Ankara 2012, 189-208.

W. H. Buckler, Lydian Records, JHS 37, 1917, 88-115.

L. Budde, Antike Mosaiken in Kilikien. 1. Frühchristliche Mosaiken in Misis-Mopsuhestia, Recklinghausen 1969 [Beiträge zur Kunst des christlichen Ostens, 5].

L. Budde, St. Pantaleon von Aphrodisias in Kilikien, Recklinghausen 1987 [Beiträge zur Kunst des christlichen Ostens, 9].

M. Cassia, Cappadocia romana. Strutture urbane e strutture agrarie alla periferia dell'Impero, Catania 2004 [Testi e studi di storia antica, 15].

J. Darrouzès (ed.), Notitiae Episcopatuum Ecclesiae Constantinopolitanae. Texte critique, introduction et notes, Paris 1981 [Géographie ecclésiastique de l'empire byzantin, I].

Gelzer - Hilgenfeld - Cuntz H. Gelzer - H. Hilgenfeld - O. Cuntz (ed.), Patrum Nicaenorum no1898 mina. Latine, Graece, Coptice, Syriace, Arabice, Armeniace. Adiecta est tabula geographica. Mit einem Nachwort von Christoph Markschies. Neudruck der 1. Auflage (1898), Stuttgart und Leipzig 1995.

${ }^{30}$ ACO II 5 77-79 ist der Brief der Bischöfe der Kappadokia II; in 77,22 wird Eustathios von Parnassos als Mitabsender genannt, in 79,13 steht seine Unterschrift.

${ }^{31}$ PG 85, 1613-1621, die Unterschrift: 1620, Z. 29-30.

${ }^{32}$ Diese Antilogie (CPG 6147) ist gegen einen nicht weiter qualifizierten Eustathios gerichtet; zur Identifikation mit Eustathios von Parnassos s. schon Tetz 1964, XLIII.

${ }^{33}$ Sollte die Datierung nicht zutreffen, ist von einem weiteren gleichnamigen Bischof auszugehen. 
Hild 1977

Hild - Hellenkemper 1990

Hild - Hellenkemper Hellenkemper-Salies 1990

Hild - Restle 1981

Honigmann 1939

Leschhorn 1993

Métivier 2005

Mitchell - Pilhofer 2019

Patac1 2016

Price - Graumann 2020

Restle 1978

Restle 1979

Russell 1987

Schäferdiek 1979
F. Hild, Das byzantinische Straßensystem in Kappadokien. Mit 114 Abbildungen auf Tafeln, 7 Abbildungen im Text und 15 Karten im Text, Wien 1977 [Veröffentlichungen der Kommission für die Tabula Imperii Byzantini, 2].

F. Hild - H. Hellenkemper, Kilikien und Isaurien. Mit 402 Abbildungen auf Tafeln, einer Abbildung im Text und drei Karten, 2 Bände, Wien 1990 [Tabula Imperii Byzantini, 5].

F. Hild - H. Hellenkemper - G. Hellenkemper-Salies, Art. Kommagene - Kilikien - Isaurien, Reallexikon zur byzantinischen Kunst IV, 1990, 182-356.

F. Hild - M. Restle, Kappadokien (Kappadokia, Charsianon, Sebasteia und Lykandos). Mit 20 Abbildungen im Text und vier Karten, Wien 1981 (Tabula Imperii Byzantini, 2).

E. Honigmann, La liste originale des pères de Nicée. A propos de l'Évêché de `Sodomar en Arabie, Byzantion XIV, 1939, 17-76.

W. Leschhorn, Antike Ären. Zeitrechnung, Politik und Geschichte im Schwarzmeerraum und in Kleinasien nordöstlich des Tauros, Stuttgart 1993 [Historia Einzelschriften, 81].

S. Métivier, La Cappadoce ( $\mathrm{IV}^{\mathrm{e}}-\mathrm{VI}^{\mathrm{e}}$ siècle). Une histoire provinciale de l'Empire romain d'Orient, Paris 2005 [Byzantina Sorbonensia, 22].

S. Mitchell - Ph. Pilhofer (eds.), From the Margins to the Mainstream. Early Christianity in Asia Minor and Cyprus, Leiden 2019 [Early Christianity in Asia Minor, 3].

S. Patacı, Some iconographic observations on a late Roman mosaic from Edessa and its contemporaneous parallels from Asia Minor and Syria, Aram 28, 2016, 429-441.

R. Price - Th. Graumann (eds.), The Council of Ephesus of 431. Documents and Proceedings. Translated, with an Introduction and Notes, Liverpool 2020 (Translated Texts for Historians, 72).

M. Restle, Art. „Kappadokien“, Reallexikon zur byzantinischen Kunst III, 1978, 968-1115.

M. Restle, Studien zur frühbyzantinischen Architektur Kappadokiens. Mit 219 Abbildungen auf Tafeln und 63 Plänen, Wien 1979 [Veröffentlichungen der Kommission für die Tabula Imperii Byzantini, 3].

J. Russell, The Mosaic Inscriptions of Anemurium. With 22 Figures and 27 Plates, Wien 1987 [ETAM, 13].

K. Schäferdiek, Wulfila. Vom Bischof von Gotien zum Gotenbischof, Zeitschrift für Kirchengeschichte 90, 1979, 107-146 (= 253292). 
Tetz 1964

Wisskirchen 2009
M. Tetz (ed.), Eine Antilogie des Eutherios von Tyana, Berlin 1964 [Patristische Texte und Studien, 19].

R. Wisskirchen, Zum „Tierfrieden“ in spätantiken Denkmälern (nach Gen. 1,29f, Jes. 11,6/8 und 65,25), Jahrbuch für Antike und Christentum 52, 2009, 142-163.

\section{Cennetteki Piskopos Eustathios: Kappadokia Parnassos'unda Bir Kilise ve Kurucusu Özet}

Tuz gölü ve Halys arasındaki Galatia bölgesinin sınırları içerisinde bulunan, uzun yıllar kaçak kazılara maruz kalan ve çeşitli tahribatlara uğrayan antik Kappadokia kenti Parnassos'ta uzun bir zamandan sonra bir kilise kazısı yapılmıştır. Bu Parnassos kentinde gerçekleştirilen ilk kazıdır. Kazıda etkileyici taban mozaikleri bulunmuş ve bu mozaik tabanın üzerine yazılmış yapı inşa yazıtı bulunan üç nefli bazilika planı olan bir kilise açığa çıkartılmıştır. Bu veriler şüphesiz Parnassos kentinin Geç Antik dönemdeki hiyerarşik durumu hakkında önemli sonuçları beraberinde getirmiştir. Kappadokia bölgesinde mozaik kullanımı çok nadirdir. Ancak bu kilisede yer alan taban mozaikleri bölgenin mozaik bakımından fakirliğine karşın oldukça etkili ve ustaca dekore edilmiştir. Ana nefin zeminini süsleyen mozaiklerde çok sayıda hayvan, bitki ve ağaçlar cennet tasviriyle birlikte yer almaktadır. Söz konusu sahne 5. yüzyıl Kilikia ve Suriye'deki yapılarda sıklıkla karşılaşılan bir motiftir. Taban mozaiğinin merkezinde Eski Yunanca bir yazıt bulunmaktadır ve bu yazıtta söz konusu kilisenin inşa süreci ile ilgili üç kişinin adı geçmektedir. $\mathrm{Bu}$ isimlerden biri de piskopos Eustathios'tur. Yazıtta ayrıca kilisenin inşası için tarih de verilmektedir. Söz konusu inşa ile ilgili pek çok yorum olsa da kilisenin 462'de inşa edilmiş olması gerektiğini öneriyoruz. Bu önerimizin nedeni ise piskopos Eustathios'un söz konusu zaman diliminde bilinen ve kristolojik tartışmaların da merkezinde yer alan bir figür olmasıdır.

Anahtar Sözcükler: Parnassoslu Eustathios, mozaik, hayvan cenneti, huzurlu krallık, inşa yazıtı, Actium erasi.

\section{Bishop Eustathios in Paradise: A church and its founder in Cappadocian Parnassos} Abstract

After years of illicit digging and demolition a church has been excavated in Parnassos of Cappadocia, situated at the border to Galatia, between Tuz Gölü and Halys. It is the first excavation in Parnassos, and a three-aisled basilica with formidable mosaics as well as a building inscription came to light, the latter giving us a glimpse of the church hierarchy in late antique Parnassos.

Mosaics are very rare in Cappadocia. The impressive and highly decorated floor of the main room depicts several animals, trees and plants in paradise. It is a motif often used in Cilicia and Syria in the 5th century. A mosaic inscription is placed in the centre, naming three men who were connected to the construction of the church, including a bishop called Eustathios. Also, a date is given as year 494 in the 15th indiction. There are several possible interpretations, but we suggest the church was built in 462 and Eustathios is to be identified with the already well-known bishop who actively participated in the theological debates of his time.

Keywords: Eustathios of Parnassus, mosaic, animal paradise, peaceful kingdom, building inscription, Actian era. 